\title{
IMPLICACIONES PALEOAMBIENTALES DE LA PRESENCIA DEL GÉNERO CERATOPHRYS (ANURA, CERATOPHRYINAE) EN CONTEXTOS ARQUEOLÓGICOS DE LA TRANSICIÓN PAMPEANO-PATAGÓNICA EN EL HOLOCENO TARDÍO (CURSO INFERIOR DEL RÍO COLORADO, ARGENTINA)
}

\author{
LUCIANA STOESSEL', SERGIO BOGAN",;"**, \\ GUSTAVO MARTÍNEZ ${ }^{\prime \cdots * *}$ Y FEDERICO L. AGNOLIN",
}

\begin{abstract}
RESUMEN
En el presente trabajo se reporta la presencia del género Ceratophrys en la localidad arqueológica San Antonio y en el sitio Paso Alsina 1, ubicados en el curso inferior del río Colorado (provincia de Buenos Aires, Argentina). Los materiales comunicados aquí son asignados al Holoceno Tardío final y representan el registro más meridional conocido para el género, encontrándose en un área de transición Pampeano-Patagónica, a más de 400 kilómetros hacia el sur de su distribución actual. Se mencionan y discuten todas las localidades holocénicas portadoras de elementos óseos referibles a Ceratophrys. La variación corológica de esta especie aquí informada es coincidente con hipótesis paleoambientales que proponen mayores índices de temperatura y posiblemente una mayor disponibilidad hídrica para el Holoceno tardío.
\end{abstract}

PALABRAS CLAVE. Argentina, curso inferior del río Colorado, transición Pampeano-Patagónica, Ceratophrys, Holoceno Tardío.

\section{PALEOEVIRONMENTAL IMPLICATIONS OF THE CERATOPHRYS GENUS (ANURA, CERATOPHRYINAE) FROM ARCHAEOLOGICAL CONTEXTS OF THE PAMPEAN-PATAGONIAN TRANSITION DURING THE LATE HOLOCENE (LOWER BASIN OF THE COLORADO RIVER, ARGENTINA)}

\section{ABSTRACT}

In this work, we report the presence of the Ceratophrys genus at the archaeological locality of San Antonio and at the Paso Alsina 1 archaeological site, situated at the lower basin of the Colorado river (Buenos Aires province, Argentina). The materials communicated here are assigned to the late

- CONICET-INCUAPA.FACSO-UNCPBA. Avda. del Valle 5737, Olavarría (B7400JWI), Argentina. lstoesse@soc.unicen.edu.ar

** Departamento Científico de Arqueología, Museo de La Plata. Paseo del Bosque, s/n (1900). La Plata, Buenos Aires, Argentina. sergiobogan@yahoo.com.ar

Fundación de Historia Natural "Félix de Azara". Departamento de Ciencias Naturales y Antropología. CEBBAD - Universidad Maimónides. Valentín Virasoro 732 (1405BDB). Buenos Aires, Argentina.

**** CONICET-INCUAPA.FACSO-UNCPBA. Avda. del Valle 5737, Olavarría (B7400JWI), Argentina. gmartine@soc.unicen.edu.ar

**:* Laboratorio de Anatomía Comparada y Evolución de los Vertebrados, Museo Argentino de Ciencias Naturales "Bernardino Rivadavia", Av. Ángel Gallardo, 470 (1405). Buenos Aires, Argentina. fedeagnolin@yahoo.com.ar 
Holocene and represent the southernmost evidence known for the genus at the Pampean-Patagonian transition, recorded more than 400 kilometers south of their present-day distribution. Additionally, we mention and discuss all the holocene localities where osseous elements referable to Ceratophrys were found. The corological variation proposed here for the genus Ceratophrys is coincident with paleoenvironmental hypotheses that propose higher temperature and a possible elevated hydric availability rates for the late Holocene.

KEY WORDS: Argentine, lower valley of Colorado River, Pampean-Patagonian transition, $\mathrm{Ce}^{-}$ ratophrys, Late Holocene.

\section{INTRODUCCIÓN}

Los escuerzos del género Ceratophrys conforman un peculiar grupo de anuros neotropicales caracterizados por su notable robustez corporal y una cabeza desproporcionadamente grande. Actualmente se consideran seis especies vivientes asignables a dicho género, de las cuales sólo dos se encuentran distribuidas en la República Argentina (Barrio 1980). En las regiones chaqueña y central de este país se distribuye la especie $C$. cranwelli Barrio 1980, mientras que una segunda especie, C. ornata (Bell 1841), restringe su distribución a la región pampeana (Lynch 1982).

En el presente artículo se reporta la existencia de una especie indeterminada del género Ceratophrys en el curso inferior del río Colorado, en una zona ecotonal, de transición PampeanoPatagónica, en contextos arqueológicos asignables al Holoceno tardío. El género no esta presente hoy en día en el área y los materiales aquí reportados representan el registro más austral para el mismo. En este sentido, se discuten y comparan espacial y cronológicamente los hallazgos del área con otros realizados en la región pampeana y se evalúan las implicaciones paleoclimáticas a partir de su presencia para el periodo bajo estudio.

Características ambientales del área de estudio

El curso inferior del río Colorado se destaca por su naturaleza alóctona y por su antiguo delta. El área de estudio se halla emplazada en el extremo sur de la Provincia Fitogeográfica del Espinal, más específicamente en el distrito del Caldén (Prosopis caldenia; Cabrera y Willink 1980) (Fig. 1). Presenta características ecológicas ecotonales, desarrollándose entre las estepas de la Provincia Pampeana al norte y la Provincia del Monte al sur (Cabrera 1976; Morello 1958). Desde el punto de vista Zoogeográfico, el área se encuentra en la Subregión Patagónica, Distrito Patagónico (Cabrera y Yepes 1960). Actualmente esta región posee un clima árido, templado y seco. Datos paleoambientales indican que las condiciones climáticas para el Holoceno medio fueron áridas, mientras que en el Holoceno tardío se acentúan condiciones semiáridas con una frecuencia mayor de lluvias que habría favorecido la expansión de lagunas durante las fases finales de este periodo (Schäbitz 1994, 2003).

Características de los sitios arqueológicos, estratigrafía y cronología.

Las investigaciones arqueológicas en el área de estudio comenzaron a desarrollarse en el año 2001 y desde entonces numerosos sitios y temáticas han sido abordadas. En este sentido, se han investigado problemáticas relacionadas a la cronología, subsistencia, tecnología lítica, asentamiento, movilidad, entierros humanos, análisis de isótopos estables y construcción de una ecología isotópica para el área, entre otras (Armentano 2004; Martínez 2008, Martínez et al. 2005; Martínez et al. 2006, 2007 a; Martínez et al. 2008; Stoessel 2007). Algunos sitios pertinentes para el tema aquí abordado son discutidos a continuación (véase Fig. 1).

\section{Localidad arqueológica San Antonio}

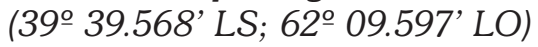

La localidad arqueológica San Antonio (compuesta por 5 sitios arqueológicos), se localiza a ca. $4 \mathrm{~km}$ de la costa atlántica, donde desemboca el río Colorado. En el sitio 1, donde se recuperaron los materiales descriptos (véase apartado siguiente), 


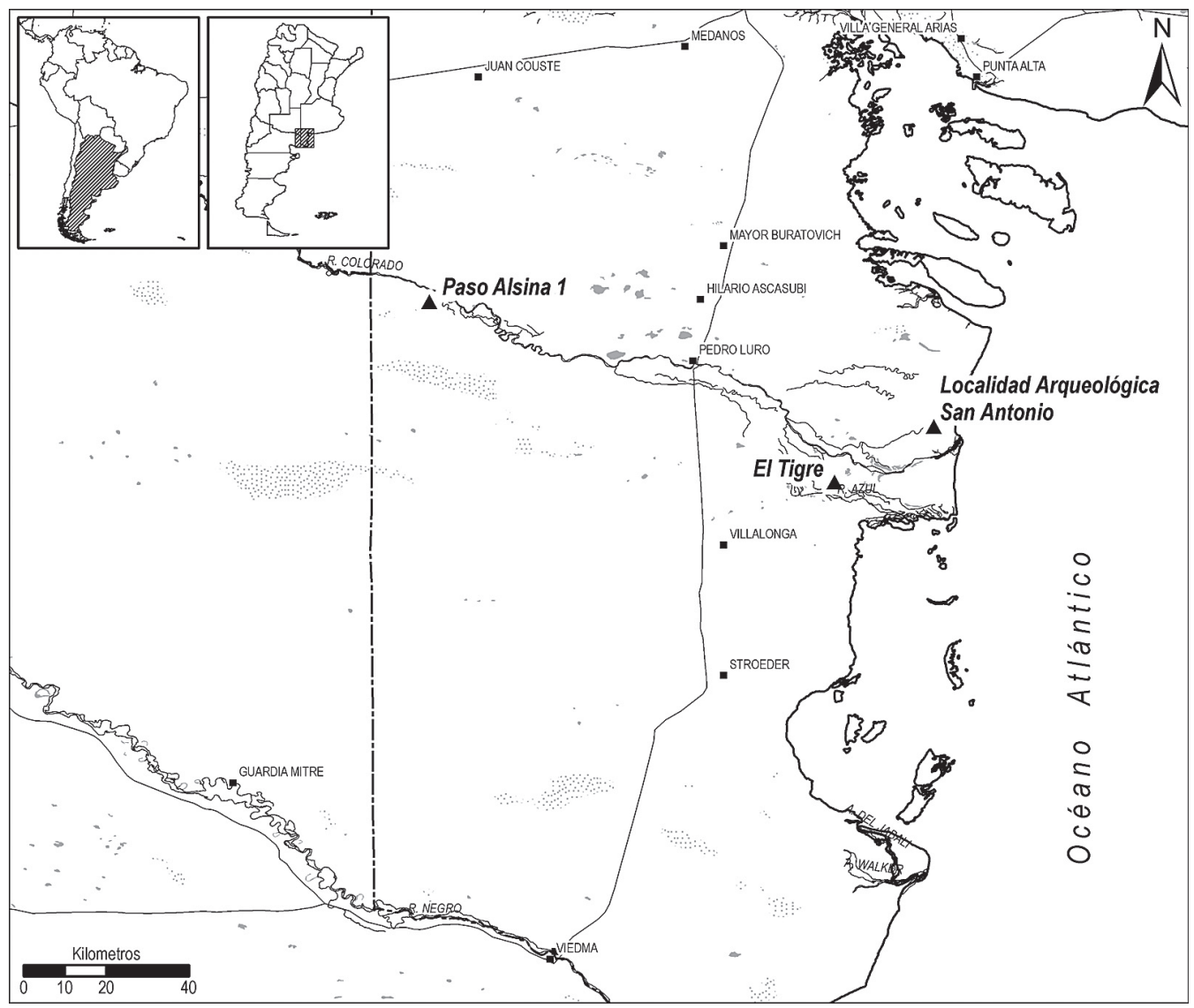

Fig. 1. Curso inferior del río Colorado. Localización de los sitios con hallazgos de Ceratophrys y otras especies discutidas en el trabajo.

se hallaron artefactos líticos, de hueso y cerámica asociados a especies terrestres, marinas y fluviales como Lama guanicoe (guanaco), Ozotoceros bezoarticus (venado de las pampas), Genidens barbus (bagre de mar), Micropogonias furnieri (corvina rubia), Percichthys sp. (perca), etc. Los restos arqueológicos fueron recuperados tanto en superficie (transectas) como en profundidad (sondeos), en un médano elongado en dirección O-E con una longitud de $200 \mathrm{~m}$ por $50 \mathrm{~m}$ de ancho, en promedio. Los estudios geoarqueológicos preliminares fueron realizados por el Dr. Gustavo Martínez (Centro de Geología de Costas, UNMDP). Se trata de una duna que se apoya sobre depósitos fluviales maduros de la antigua llanura de inundación del río Colorado. Así, los depósitos originales del médano, donde se encuentra el material arqueológico, son exclusivamente de origen eólico. La duna se encuentra a ca. $90 \mathrm{~m}$ del cauce del río Colorado nuevo.
En la secuencia estratigráfica definida en base a dos sondeos realizados sobre los flancos $\mathrm{N}$ y S de la hoyada de deflación se registraron 5 unidades principales. Las unidades 5 (arena arcillo-limosa, antigua llanura de inundación) y 4 (arena), estériles arqueológicamente, se encuentran en la parte basal del depósito. Los artefactos líticos, faunísticos, cerámicos, etc. se localizan por encima de ambas, en las unidades 2 y 3 (arenas con raíces y bioturbaciones). Finalmente, hacia la parte cuspidal del perfil, la unidad 1 (Horizonte A actual) no posee asociaciones artefactuales. Claramente, el componente arqueológico se relaciona a las unidades 2 y 3 . Las distribuciones de artefactos y restos faunísticos en superficie son producto de la deflación que ha actuado sobre un flanco de la duna, afectando a las unidades superiores. La localización de los artefactos y restos faunísticos es el producto de ocupaciones indígenas que tuvieron lugar sobre la superficie de 
la duna en algún momento entre el ca. 1.500-400 años AP, a juzgar por la presencia de cerámica (véase Martínez 2008). En consecuencia, los especimenes óseos correspondientes a Ceratophrys registrados en el sitio indican que esta especie, hoy ausente en el área, habría habitado la misma al menos durante el lapso 1.500-400 años AP. A continuación se describe el material de Ceratophrys recuperado y sus características contextuales.

1) Muestreo № 3, material superficial. Premaxilar izquierdo completo y fragmento de maxilar derecho. (FCS.SA1.M3.590.1) y (FCS.SA1.M3.590.2). (Fig. 2C-D y E).

2) Sondeo 1, Nivel 3 (0,60-0,70 m). Esfenoetmoides incompleto (FCS.SA1.S1.486.1). (Fig. $2 \mathrm{~F})$.

3) Sondeo 1, Nivel 7 (1,00-1,10 m). Fragmento posterior de frontoparietal izquierdo (FCS.SA1. S1.506.1). (Fig 2 A).

4) Sondeo 1, Nivel 10 (1,30-1,40 m). Frontoparietal derecho (FCS.SA1.S1.525.1). (Fig. 2 B).

5) Sondeo 1, Nivel $11(1,40-1,50)$. Frontoparietal izquierdo (FCS.SA1.S1.534.1).

A excepción del espécimen recuperado en superficie, los restantes elementos óseos provienen de las Unidades 2 y 3 , donde se localizó la totalidad del componente arqueológico.

Sitio Arqueológico Paso Alsina 1 (39은 23.277' LS; 63‥ 15.360' LO)

En el sitio Paso Alsina 1, hacia el final de una cadena medanosa, se recuperaron diez entierros humanos de modalidad secundaria dispuestos en un área pequeña $\left(\mathrm{ca} .6 \mathrm{~m}^{2}\right)$. Sobre la base de 13 fechados radiocarbónicos provenientes de los entierros se estimó una media ponderada de $483 \pm 20$ años AP (véase Martínez et al. 2007 a y Martínez 2008). El espécimen registrado de Ceratophrys se halló en asociación con el entierro 1 , en los niveles inferiores del mismo, en un área de transición arenosa y de depósitos fluviales, hacia la base este entierro. En consecuencia, dada la cronología del sitio, se estima que este género estuvo presente en el área en ca. 500 años AP. El espécimen reportado corresponde a: 1) Maxilar izquierdo completo (FCS. PA1.E1.P4.1) (Fig. 2 G-H).
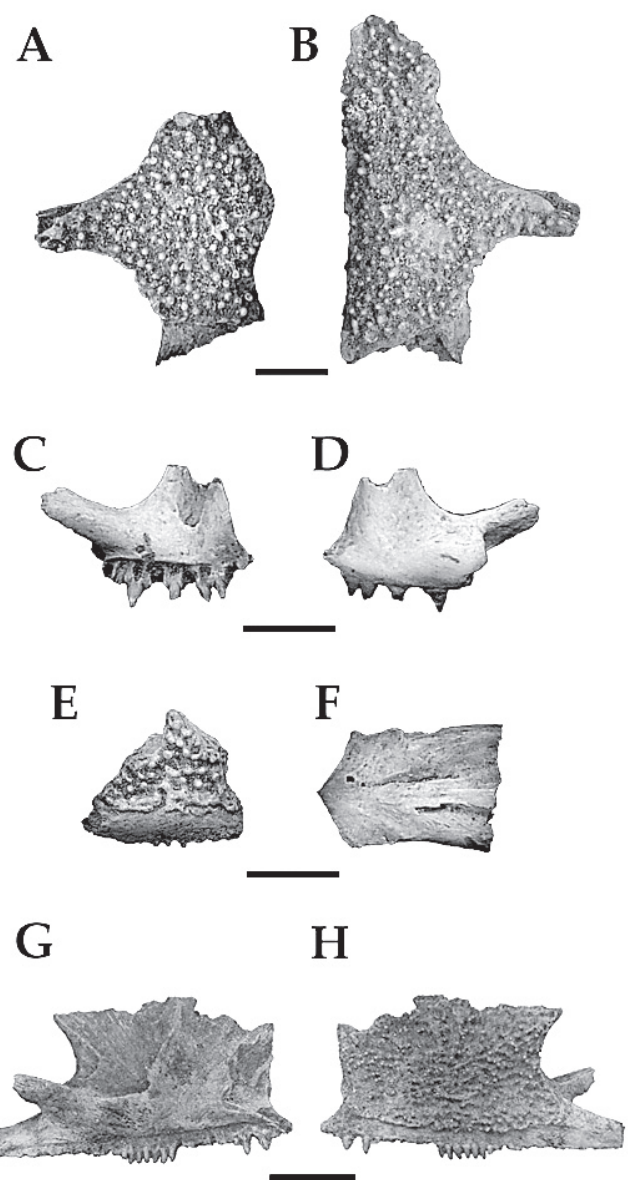

Fig. 2. Ceratophrys sp. (A) fragmento posterior de frontoparietal izquierdo en vista dorsal (FCS.SA1. S1.506.1); (B) frontoparietal derecho en vista dorsal (FCS. SA1.S1.525.1); Premaxilar izquierdo en vistas (C) interna

(D) externa (FCS.SA1.M3.590.1); (E) maxilar derecho incompleto en vista externa (FCS.SA1.M3.590.2); (F) esfenoetmoides en vista palatal (FCS.SA1.S1.486.1); Maxilar izquierdo completo en vistas $(\mathrm{G})$ interna, y $(\mathrm{H})$ externa (FCS.

PA1.E1.P4.1). A-F: Escala, 5 mm; G-H: Escala, 10 mm.

Sistemática, hábitat y antecedentes de hallazgos de Ceratophrys sp.

La sistemática del género referido es la siguiente:

Anura Rafinesque, 1815

Neobatrachia Reig, 1958

Ceratophryinae Tschudi, 1838

Ceratophrys Wied-Neuwied, 1824

Ceratophrys sp. 
Los materiales aquí registrados son referibles a Ceratophryinae sobre la base de la siguiente combinación de caracteres: 1) dientes no pedicelados, muy calcificados y anquilosados a los huesos, 2) huesos craneanos (a excepción del premaxilar) hiperosificados y fuertemente esculturados, 3) ausencia de repisa palatal en el premaxilar y maxilar (Evans et al. 2008; Fabrezi 2001). Adicionalmente, los materiales pueden ser referidos al género Ceratophrys debido a los siguientes caracteres derivados: 1) huesos dérmicos craneanos con una fuerte ornamentación de tipo tuberculada, 2) presencia de dos escotaduras óticas en el dermocráneo, 3) región esfenoetmoidal fuertemente osificada, 4) proceso alar del premaxilar no diferenciado de la cara facial del hueso (Agnolin 2005; Peri 1993a). Dentro del género Ceratophrys la identificación a nivel especifico de materiales óseos aislados es hasta el momento poco confiable (Peri 1993b). En consecuencia, la naturaleza de los materiales aquí presentados impide una asignación específica precisa.

El registro más austral para el género Ceratophrys lo representa la especie extinta C. (Stombus) ameghinorum Fernícola 2001, procedente del Plioceno de la localidad de Farola de Monte Hermoso, al sur de la provincia de Buenos Aires (Fernícola 2001). Adicionalmente, la presencia de diversos especímenes del género Ceratophrys ha sido indicada para numerosas localidades del Plioceno y Pleistoceno de la provincia de Buenos Aires (Agnolin 2005; Cione y Báez 2007).

Las menciones de escuerzos para el Holoceno de la provincia de Buenos Aires son muy escasas, $y$ se restringen a unas pocas localidades ubicadas principalmente en el litoral marítimo del sur de la provincia. En capas de arcillas lacustres, emplazadas en el sector intermareal del Camping Americano en el Municipio de Monte Hermoso, Pardiñas (2001) reporta restos craneales (MMH 85-3-8 yMMH 852-11) y postcraneales (MMH 90-2-1 y MMH 8-2-5) referibles a Ceratophrys cf. C. ornata. Los depósitos portadores de este material presentan una cronología entre 8 y 9 ka y se hallan inmediatamente por debajo del nivel arqueológico con icnitas humanas del sitio Monte Hermoso I (Pardiñas 2001) (véase Fig. 3). Para el sitio arqueológico Laguna Tres Reyes, ubicado en el partido de Gonzales Chávez, se reportan dos cráneos (MLP 86.VIII.1.4 y MLP 86.VIII.1.5/6) del Holoceno Temprano referidos a Ceratophrys sp. (Peri 1993 a; Cione y Báez 2007; véase también Salemme y Madrid
2007). En niveles correspondientes al Holoceno Temprano del arroyo Tapalqué (Olavarría), se colectaron dos cráneos (MLP 86.III.25.150 y MLP 86.III.25.151) interpretados como una forma tetraploide y a otra octoploide, ambas de estatus específico indeterminado (Mercadal de Barrio y Barrio 2002). En el sitio arqueológico Fortín Necochea (partido de General La Madrid, provincia de Buenos Aires) se reporta un cráneo referible a este género (MLP 96.V.18.12; Mercadal de Barrio y Barrio 2002), relacionado cronológicamente al Holoceno Temprano-Medio. Para el "Aimarense" (Holoceno Temprano) de la localidad marítima de Miramar, ha sido colectado un cráneo completo referido por Frenguelli (1921) a la especie viviente Ceratophrys ornata. Recientemente, han sido mencionados elementos craneanos asignados a esta misma especie para la Bahía Samborombón, partido de General Lavalle (Aldazabal et al. 2007). Dicho material fue recuperado en el sitio el Divisadero Monte 6 en asociación con un depósito antrópico del Holoceno Tardío (Aldazabal et al. 2007) y consiste en un maxilar derecho y premaxilar izquierdo, que presentan la siguiente combinación de caracteres que indican su pertenencia a Ceratophrys: 1) maxilar fuertemente esculturado con tubérculos y 2) proceso alar del premaxilar no diferenciado de la cara facial del hueso (véase más arriba). Sin embargo, la naturaleza fragmentaria del material registrado en la Bahía Samborombón impide una asignación específica precisa, y consecuentemente estos restos son reidentificados como Ceratophrys sp.

Tanto C. ornata como C. cranwelli se reproducen en desbordes de lagunas, charcas estacionales, etc., siendo el agua un factor ecológico limitante para la puesta de huevos y el normal desarrollo de los individuos juveniles de estos escuerzos. El hábitat de ambas especies es diferente tanto en la fisonomía vegetal como en las condiciones climáticas. La especie chaqueña $C$. cranwelli se encuentra distribuida en regiones subtropicales secas con clima continental caracterizado por una gran cantidad de radiación solar y baja humedad atmosférica (Barrio 1980). En cuanto a la fisonomía vegetal, esta especie habita la Provincia Fitogeográfica Chaqueña y parte del Espinal, más comúnmente bosques secos y arbustales. Por otro lado, la especie $C$. ornata se circunscribe a la Provincia Fitogeográfica Pampeana, y al igual que el escuerzo chaqueño habita ambientes abiertos y pobremente arbolados (Fig. 3). 


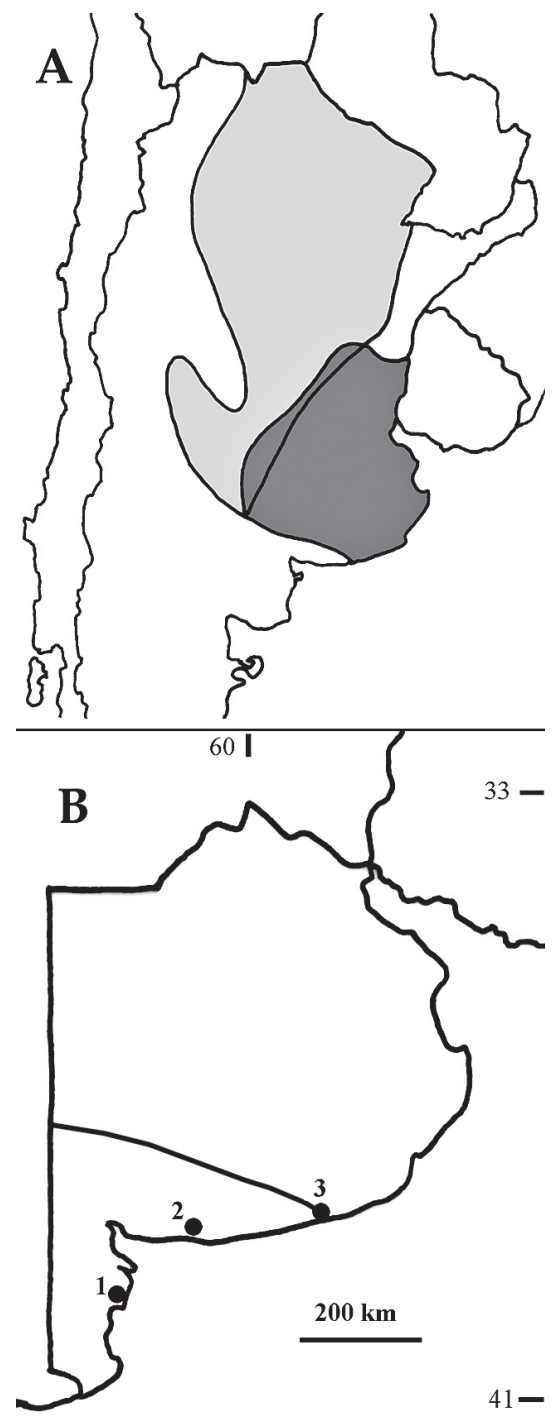

Fig. 3. A: Distribución actual de Ceratophrys ornata (tono oscuro) y Ceratophrys cranwelli (tono claro). B: Localidades holocénicas con hallazgos de Ceratophrys sp. fuera del área de distribución actualmente conocida para el género: (1) San Antonio 1 y Paso Alsina 1; (2) Camping Americano en el Municipio de Monte Hermoso. (3) Necochea, localidad más meridional actualmente registrada para el género Ceratophys.

Los escuerzos actuales de la especie $C$. ornata representan el registro más austral para el género, remontándose hasta la localidad balnearia de Necochea, al sur de la provincia de Buenos Aires (Barrio 1980; Lynch 1982) (véase Fig. 3). La distribución de esta especie se encuentra actualmente restringida a regiones caracterizadas por un clima templado-cálido (temperaturas medias anuales que varían de 13 a $17^{\circ} \mathrm{C}$ ), con baja amplitud térmica estacional (debido a la fuerte influencia marítima) y con lluvias anuales que varían de 600 a 1200 mm anuales (Barrio 1980).

Actualmente, el curso inferior del río Colorado, se caracteriza por un clima árido, templado y seco, con una temperatura media anual de $12^{\circ} \mathrm{C}$. Las precipitaciones medias anuales actuales son de 466 mm (Sánchez et al. 1998). Para el Holoceno tardío (últimos 3.000 años AP), Schabitz (2003:298) infiere precipitaciones de $260-380 \mathrm{~mm}$ anuales. Estos valores son inferiores a aquellos tolerados actualmente por el género Ceratophrys o por cualquier otro Ceratophryinae conocido (Barrio 1980). Posiblemente, la presencia de Ceratophrys en el Holoceno Tardío en las proximidades del río Colorado pueda vincularse con mayores temperaturas y con una mayor disponibilidad hídrica en la región (véase discusión más abajo).

\section{DISCUSIÓN Y CONSIDERACIONES FINALES}

El estado de avance en el estudio de los procesos de formación de sitios es preliminar, aunque de acuerdo a la cronología relativa y absoluta es posible inferir en qué momento se incorporaron al registro arqueológico de San Antonio 1 y Paso Alsina 1 los especimenes óseos del género $\mathrm{Ce}$ ratophrys. Considerando la ausencia del género en el área en la actualidad se realizan las siguientes consideraciones. En una escala temporal de grano grueso, como se discutió anteriormente, los restos arqueológicos de San Antonio 1 se habrían depositado entre ca. 1.500-400 años AP. Sin embargo, este lapso temporal puede ser aun más acotado. Un fechado radiocarbónico proveniente del sitio 2 de la localidad San Antonio (sondeo 1) arrojo un resultado de $764 \pm 45$ años $\mathrm{AP}\left(\delta \mathrm{C}^{13}-20,9 \%\right.$; AA-77966; hueso de guanaco). En consecuencia, de acuerdo a la homogeneidad de los contenidos artefactuales de los sitios que componen la localidad San Antonio (e.g., sitio 1) y a la cronología del sitio Paso Alsina 1, es posible que los especimenes óseos se hayan depositado durante el lapso de ca. 800-400 años AP.

El registro de Ceratophrys en el Holoceno Tardío final en el curso inferior del río Colorado es concordante con el hallazgo de géneros que 
poseen similares requerimientos ambientales, como es el caso del roedor sigmodontino semiacuático Holochilus brasiliensis en el sitio El Tigre (G. Moreira com. pers. 2006), con una cronología de ca. 1.000-500 años AP (Martínez et al. 2005; Martínez et al. 2007 b), y en los sitios Angostura 1 (ca. 900-800 años AP) y Negro Muerto (500-400 años AP), ubicados en el curso medio del río Negro (Prates 2008:278). Actualmente, Holochilus brasiliensis presenta como limite sud oriental el eje Chasico-Bahía Blanca (Pardiñas et al. 2004).

Las reconstrucciones paleoclimáticas propuestas para los últimos 1.000 años AP en el Hemisferio Sur han sido variables (e.g., Mancini y Trivi de Mandri 1992: Stine y Stine 1990; Villalba 1994a, 1994b). En general, se han reconocido básicamente dos eventos climáticos principales: la Anomalía Climática Medieval (ACM; 1.200-800 AP) y la Pequeña Edad de Hielo (PEH; 450-200 AP). Las implicancias paleoclimáticas de estos dos eventos sugieren episodios persistentes de calentamiento $y$ enfriamiento, aunque variables temporalmente cuando se consideran escalas espaciales amplias. En este sentido, factores locales y regionales producen significativas diferencias respecto, por ejemplo, de las precipitaciones (Soon et al. 2003). En el caso de Patagonia se propuso que mientras en el norte se producirían condiciones secas y cálidas entre ca. 1.080-1.250 AD, en el sur existirían condiciones frías con exceso de precipitaciones entre el ca. 1.050-1.200 AD (patrón bipolar sensu Agosta et. al. 2005; véase Villaba 1990, 1994; Stine 1994). En consecuencia, la expresión paleoclimática de estos eventos en escalas areales puede presentar variaciones. Algunos autores (Carignano 1999; Cioccale 1999; Iriondo 1999), plantean que durante la Anomalía Climática Medieval (ACM u Optimo Medieval) las condiciones climáticas habrían sido más cálidas y húmedas. Sin embargo, por lo expresado anteriormente, la existencia simultánea de estos dos factores es controvertida (véase discusión en Tonni 2003 y Agosta et al. 2005). La presencia de los géneros Ceratophrys y Holochilus en la transición Pampeano-Patagónica es compatible, al menos, con la existencia de pulsos cálidos en el área durante el periodo sugerido para la ACM. Por el contrario, con el advenimiento de las condiciones propias de la Pequeña Edad de Hielo (e.g., frío y árido) estas especies se habrían visto fuertemente constreñidas. Independientemente de la existencia en el norte de Patagonia de condiciones húmedas durante la ACM, es posible que las especies referidas ocuparan estos espacios debido a que las mismas hayan estado sujetas a una dispersión ocurrida a través de importantes corredores fluviales (e.g., ríos Colorado y Negro). En este sentido, los cuerpos de agua pueden modificar localmente las condiciones áridas favoreciendo el ingreso de fauna de estirpes no locales (e.g., brasílicas) (Pardiñas 1999; Quattrocchio et al. 2008). Asimismo, franjas medanosas litorales con condiciones edáficas y microclimáticas especificas pueden actuar como vías de ingreso de ciertas especies (Pardiñas et al. 2004). Estas situaciones se verían favorecidas por la situación ecotonal del área que la hace muy sensible a los cambios paleoclimáticos (Quattrocchio et al. 2008:135) y que actúa como una bisagra zoogeográfica (Pardiñas et al. 2004:226).

La información paleoclimática actual para el área de transición Pampeano-Patagónica ligada al litoral Atlántico es aún insuficiente para demostrar la existencia de eventos relacionados a la ACM o a la PEH. Sin embargo, la evidencia discutida en este trabajo a partir de la distribución de anuros como Ceratophrys y de roedores como Holochilus brasiliensis indica que condiciones climáticas más cálidas y probablemente más húmedas habrían tenido lugar entre ca. 1.000-400 años AP en el sector comprendido por los ríos Colorado y Negro.

\section{AGRADECIMIENTOS}

Este trabajo fue posible gracias a subsidios provistos por la Fundación Antorchas (Proyecto 14022-2), CONICET (PIP 6147/05) y ANPCyP (PICT № 264-06) asignados a uno de los autores (G.M.). Agradecemos el apoyo institucional del INCUAPA (FACSO, UNCPBA). Al Dr. G.A. Martínez (Centro de Geología de Costas, UNMdPA) por la interpretación geoarqueológica en San Antonio y a Germán Moreira (UNLP) por la determinación de Holochilus en el sitio El Tigre. Agradecemos a Flavia Zorzi por las importantes sugerencias gramaticales. Asimismo, agradecemos especialmente a Gabriel L. Lio su ayuda en la confección de las imágenes. 


\section{BIBLIOGRAFÍA}

AGNOLIN, F.L. 2005. Una nueva especie del género Ceratophrys (Anura; Ceratophryidae) del Pleistoceno Inferior-Medio de la provincia de Buenos Aires. Studia Geologica Salmanticensia 41: 45-55.

AGOSTA, E.A., C. FAVIER DUBOIS y R.H. COMPAGNUCCI 2005. Anomalías climáticas en la patagonia durante el Calentamiento Vikingo y la Pequeña Edad de Hielo. Trabajo publicado en el Libro del Congremet. Buenos Aires.

ALDAZABAL, V., M. SILVEIRA y E. EUGENIO 2007. Zooarqueologia del sitio "El Divisadero Monte 6" (Partido de General Lavalle, Provincia de Buenos Aires). Libro de resúmenes XVI Congreso Nacional de Arqueología Argentina. Tomo III: 241-246. Jujuy.

ARMENTANO, G. 2004 Organización de la Tecnología Lítica en el valle inferior del río Colorado (Pdos. de Patagones y Villarino, Pcia. de Buenos Aires). Tesis de Licenciatura inédita, FACSO-UNCPBA. Olavarría. MS.

BARRIO, A. 1980. Una nueva especie de Ceratophrys (Anura, Ceratophryidae) del Dominio Chaqueño. Physis 39: 21-30.

CABRERA, A. 1976. Regiones Fitogeográficas Argentinas. Enciclopedia Argentina de Agricultura y Jardinería. Tomo II (1). Editorial Acme. Buenos Aires.

CABRERA, A.L. y J. YEPES 1960. Mamíferos Sudamericanos. Tomo I. EDIAR. Buenos Aires.

CABRERA, A.L. y A. WILLINK 1980. Biogeografía de América Latina. Monografía del Departamento de Asuntos Científicos y Tecnológicos de la Secretaría General de la Organización de los Estados Americanos. Pp. 122.

CARIGNANO, C.A. 1999. Late Pleistocene to recent climate change in Cordoba Province, Argentina: Geomorphological Evidence. Quaternary International 57-58: 117-134.

CIOCCALE, M.A. 1999. Climatic fluctuations in the central region of Argentina in the last 1000 years. Quaternary International 62: 35-47.

CIONE, A.L. y A.M. BAEZ 2007. Peces continentales y anfibios cenozoicos de Argentina: los últimos cincuenta años. Ameghiniana, Publ. Especial, 11: 195-220.

EVANS, S.E., M.E.H. JONES y D.W. KRAUSE 2008. A giant frog with South American affinities from the Late Cretaceous of Madagascar. Proceedings of the National Academy of Sciences 105: 2951-2956.

FABREZI, M. 2001. Variación morfológica de la dentición en anuros. Cuadernos de Herpetología 15: 17-28.
FERNÍCOLA, J.C. 2001. Una nueva especie de Ceratophrys (Anura, Leptodactylidae) en el Neógeno de la provincia de Buenos Aires, Argentina. Ameghiniana 38 (4): 385-391.

FRENGUELLI, J. 1921. Los terrenos de la costa atlántica en los alrededores de Miramar (prov. de Buenos Aires) y sus correlaciones. Boletín de la Academia Nacional de Ciencias de Córdoba 24: 325-485.

IRIONDO, M. 1999. Climatic changes in the South American plains: Records of a continent-scale oscillation. Quaternary International 57-58: 93-112.

LYNCH, J.D. 1982. Relationships of the frogs of the genus Ceratophrys (Leptodactylidae) and their bearing on hypothesis of Pleistocene forest refugia in South America and punctuated equilibria. Syst. Zool., 31: 166-179.

MANCINI, M.V. y M.E. TRIVIDE MANDRI 1992. Vegetación en el área Río pinturas. Análisis polínico del Alero Cárdenas. En Contribución a la Arqueología del Río pinturas, provincia de Santa Cruz. Editorial búsqueda de Ayllu (Concepción del Uruguay), pp. 193-201. Buenos Aires

MARTÍNEZ, G.A. 2008. Arqueología del curso inferior del río Colorado: Estado actual del conocimiento. En INCUAPA 10 años: Perspectivas en Arqueología Pampeana Contemporánea, editado por G. Politis. Facultad de Ciencias Sociales de Olavarría, Universidad Nacional del Centro de la Provincia de Buenos Aires, en prensa.

MARTÍNEZ G.A., A.F. ZANGRANDO y L. STOESSEL 2005. Sitio El Tigre (Pdo. De Patagones, Pcia. de Buenos Aires, Argentina): evidencias sobre la explotación de peces en el curso inferior del Río Colorado e implicaciones para los sistemas de subsistencia. Magallania 33 (2): 99-114

MARTÍNEZ, G., P. BAYALA, G. FLENSBORG y R. LÓPEZ. 2006. Análisis Preliminar de los entierros humanos del sitio Paso Alsina 1 (Pcia. de Buenos Aires). Intersecciones en Antropología 7: 95-108.

MARTÍNEZ, G., G. FLENSBORG, P. BAYALA y R. LÓPEZ. 2007 a. Análisis de la composición anatómica, sexo y edad de dos entierros secundarios del sitio Paso Alsina 1 (Pdo. de Patagones, Pcia. de Buenos Aires). En: Arqueología en las Pampas, editado por C. Bayón, A. Puppio, M.I. González, N. Flegenheimer y M. Freire,Tomo I, pp. 41-58. Sociedad Argentina de Antropología. Buenos Aires.

MARTÍNEZ, G., L. STOESSEL y G. ARMENTANO. 2007 b. Cronología, procesos de formación y ocupaciones humanas en el sitio El Tigre (Pdo. de Patagones, Pcia. de Buenos Aires). Trabajo presentado en la Mesa de Comunicaciones de Patagonia. XVI Congreso Nacional de Arqueología Argentina. San Salvador de Jujuy. 
MARTÍNEZ, G., A.F. ZANGRANDO y L. PRATES. 2008. Isotopic ecology and human paleodiets in the lower basin of the Colorado River (Buenos Aires province, Argentina). Trabajo aceptado para su publicación en International Journal of Osteoarchaeology.

MERCADAL DE BARRIO, I.T. y A. BARRIO. 2002. Tetraploidía en Ceratophrys (Anura, Leptodactylidae), análisis del registro fósil. Iheringia, 92: 17-31.

MORELLO, J. 1958. La Provincia Fitogeográfica del Monte. Opera Lilloana 2: 11-155.

PARDIÑAS, U.F.J. 1995. Novedosos cricétidos (Mammalia, Rodentia) en el Holoceno de la región pampeana, Argentina. Ameghiniana 32: 197-203.

PARDIÑAS, U.F.J. 1999. Los roedores muroideos del Pleistoceno Tardío-Holoceno en la región pampeana (sector este) y Patagonia (República Argentina): aspectos taxonómicos, importancia bioestratigráfica y significación paleoambiental. Tesis Doctoral, Facultad de Ciencias Naturales y Museo, Universidad Nacional de La Plata.

PARDIÑAS, U.F.J. 2001. Condiciones áridas durante el Holoceno Temprano en el sudoeste de la provincia de Buenos Aires (Argentina): vertebrados y tafonomía. Ameghiniana, 38: 227-236.

PARDIÑAS, U.F.J., A.M. ABBA y M.L. MERINO. 2004. Micromamíferos (Didelphimorphia y rodentia) del sudoeste de la provincia de Buenos Aires (Argentina): taxonomía y distribución. Mastozoología Neotropical 11 (2): 211-232.

PERÍ, S.I. 1993a. Relaciones evolutivas de las especies de la Subfamilia Ceratophryinae (Anura: Leptodactylidae). Tesis doctoral, Facultad de Ciencias Naturales y Museo de La Plata, 223p. Inédita.

PERÍ, S.I. 1993b. Ceratophrys (Anura, Leptodactylidae) en el Holoceno de Laguna Los Tres Reyes, provincia de Buenos Aires, Argentina. Ameghiniana 31 (1): 3-7.

PRATES, L. 2008. Los indígenas del río Negro. Un enfoque arqueológico. Colección Tesis Doctorales, Sociedad Argentina de Antropología.

QUATTROCCHIO, M.E., A.M. BORROMEI, C.M. DESCHAMPS, S.C. GRILL y C.A. ZABALA. 2008. Landscape evolution and climate changes in the Late Pleistocene-Holocene southern Pampa (Argentina): Evidence from palynology, mammals and sedimentology. Quaternary Internacional 181: $123-138$.
SALEMME, M.C. y P.E.E. MADRID. 2007. The archaeofaunas from Laguna Tres Reyes 1 site: taxonomic richness and abundance during the beginning of the late Holocene in the South-east Pampean Region (Argentina). En: Taphonomy and Zooarchaeology in Argentina, pp. 121-142. Editado por M.A. Gutiérrez, L. Miotti, G. Barrientos, G. Mengoni Goñalons y M. Salemme. BAR International Series 1601. Archaeopress, Oxford.

SÁNCHEZ, R., N. PEZZOLA y J. CEPEDA 1998. Caracterización edafoclimática del área de influencia del INTA. EEA Hilario Ascasubi. Boletín de Divulgación 18, INTA. Buenos Aires.

SCHÄBITZ, F. 1994. Holocene climatic variations in northern Patagonia, Argentina. Palaeogeography, paleoclimatology, palaeoecology 109: 287-294.

SCHÄBITZ, F. 2003. Estudios polínicos del Cuaternario en las regiones áridas del sur de Argentina. Revista del Museo Argentino de Ciencias Naturales 5 (2): 291-299.

SOON, W., S. BALIUNAS, C. IDSO, S. IDSO y D.R. LEGATES 2003. Reconstructing climatic and environmental changes of the past 1000 years: a reappraisal. Energy and Environment (14) 2 y 3: 293-296.

STINE, S. 1994. Extreme and persistent drought in California and Patagonia during Medieval Time. Nature 369: 546-549.

STINE, S. y M. STINE 1990. A record from lake Cardiel of climatic change in southern South America. Nature 345: 705-708.

STOESSEL, L. 2007. Análisis arqueofaunísticos de los sitios Loma Ruíz 1 y El Tigre (partidos de Villarino y Patagones, provincia de Buenos Aires). Aportes para el conocimiento de la subsistencia en el valle inferior del río Colorado durante el Holoceno tardío. Intersecciones en Antropología 8: 235-251.

TONNI, E.P. 2003. Faunas y Clima en el Cuaternario de la Mesopotamia Argentina. Miscelánea 12: 5-12.

VILLALBA, R. 1990. Climatic Fluctuations in Northern Patagonia during the Last 1000 Years as inferred from Tree-Ring Records. Quaternary Research 34: 346-360.

VILLALBA, R. 1994a. Fluctuaciones climáticas en latitudes medias de América del sur durante los últimos 1000 años: sus relaciones con la oscilación del sur. Revista Chilena de Historia Natural 67: 453-461.

VILLALBA, R. 1994b. Tree-ring and glacial evidence for the Medieval Warm Epoch and the Little Ice Age in Southern South America. Climatic Change 26: 183-197. 
